\title{
Four Images from Apparent Position (2012)
}

\author{
PALOMA POLO
}

SPANISH VISUAL ARTIST

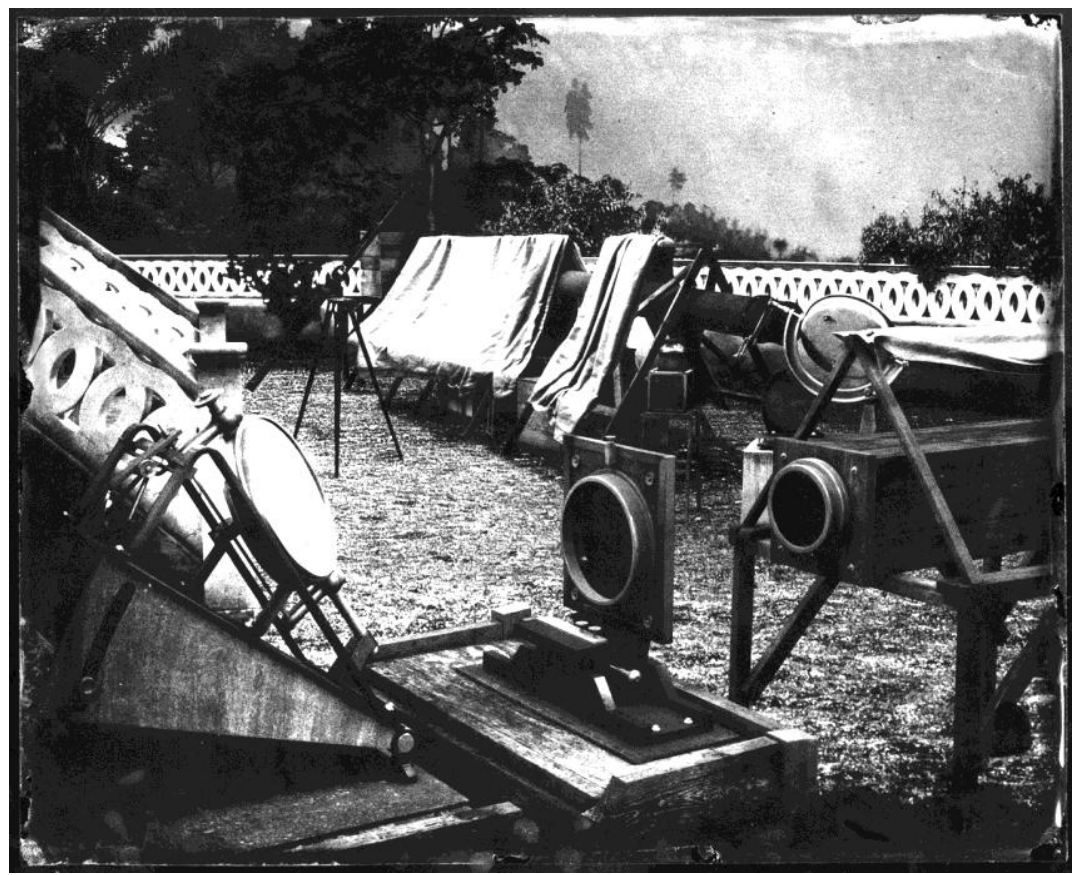

Budhi: A Journal of Ideas and Culture 17.2 (2013): 169-74. 


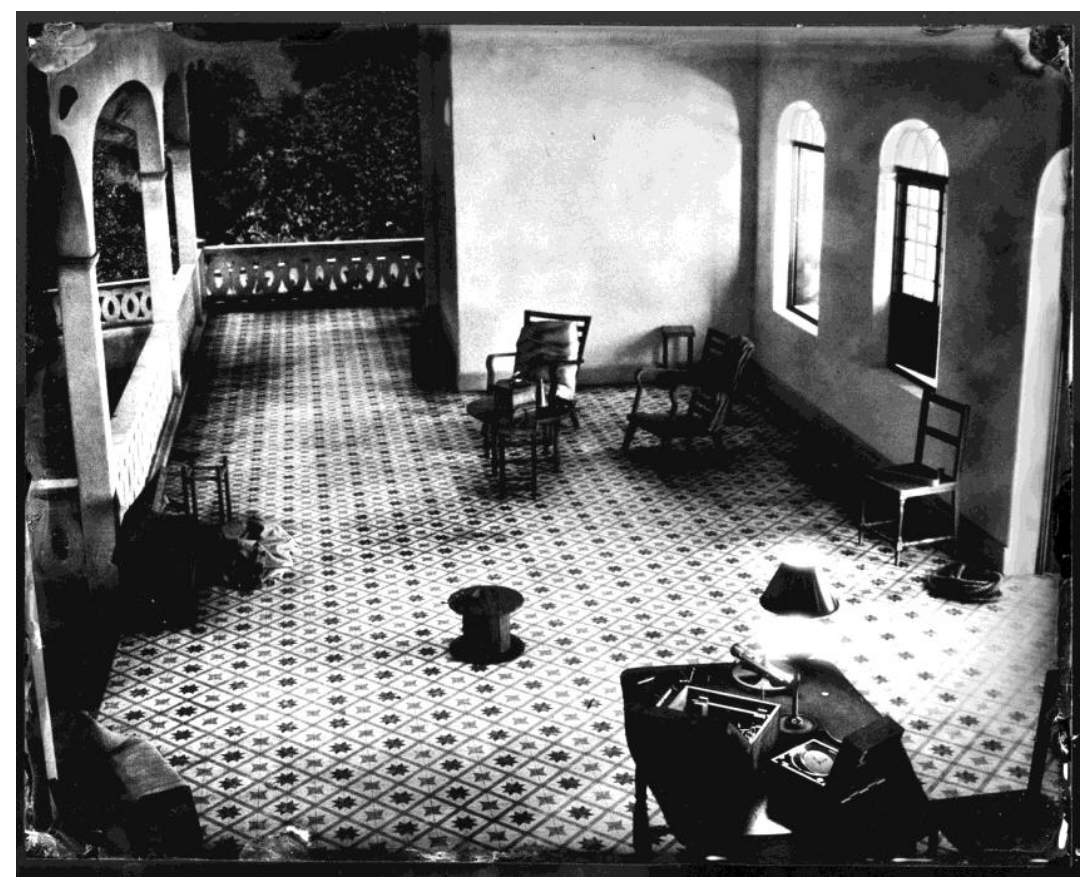




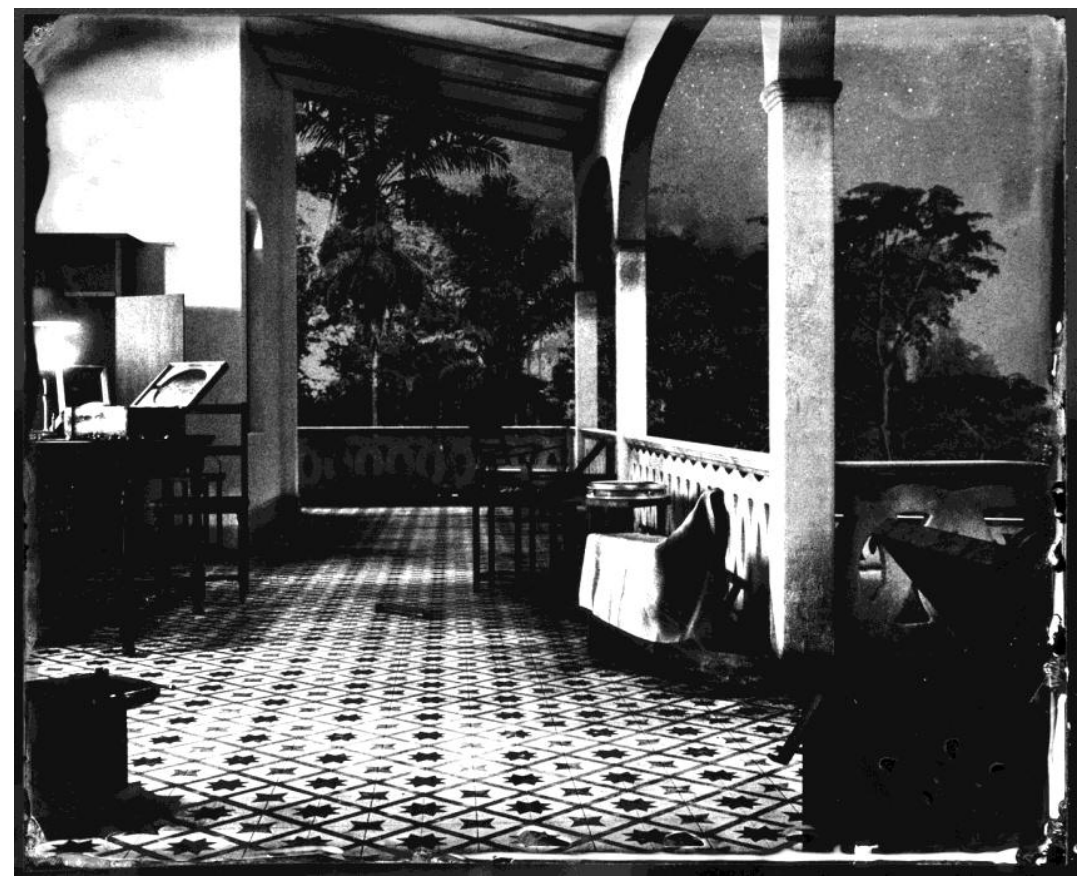




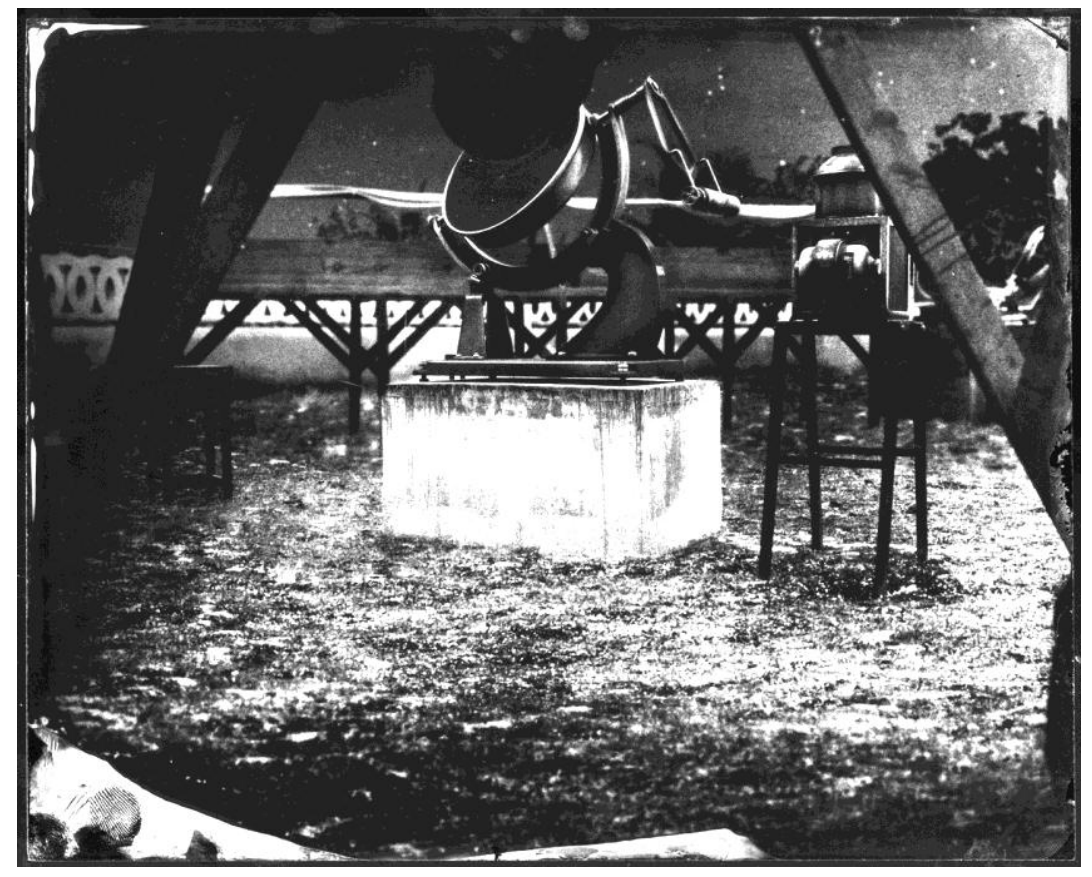


Paloma Polo's project at the Museo Reina Sofía emerged from research into expeditions undertaken in various parts of the world during the colonial era that sought to observe and document astronomical phenomena.

At first glance, her work addresses issues related to the connection between scientific knowledge and the imperialist projects of the European powers in the 19th and 20th centuries, but Apparent Position also gives rise to less immediate reflections and relations between events that seem to intersect at a given time and place, in the manner of an eclipse.

The starting point is a historically and geographically verifiable event: Sir Arthur Eddington's 1919 expedition to the island of Príncipe, a Portuguese colony in the Gulf of Guinea, to observe the effects of a total solar eclipse. Although there are precise reports on the calculations and conclusions of the expedition, there are no photographic records of the experience. Only a stone stele mounted upon a whitewashed plinth at the approximate spot where the eclipse was observed reminds us that Eddington's achievement signified the verification of Albert Einstein's theory of relativity.

Eddington's expedition does not appear to have been the touchstone in the verification of Einstein's theory. Later research indicates that its scientific results were rather poor, and although Eddington's expedition "officially" legitimized the validity of the theory of relativity, such confirmation actually came only two years later.

Paloma Polo's proposal is presented in three formats: a 16-mm film transferred to digital video, photographs on glass, and a printed book.

The images depict a silent scene that might well have taken place at the time of the expedition-photographs on glass illustrating the appearance of the expedition's surroundings during the eclipse. As Einstein pointed out, numerous variables enter into play: the peculiar light of an eclipse; the procedure for making a printed record of it with a photographic camera; and the constructed nature of such images. The images in question are virtual reconstructions of a space from the standpoint of the present. They seek to recreate as exactly as possible the look of the instruments, the furnishings, the decoration, and the architectural features of the epoch under the momentary penumbra of the eclipse, all recreated with the 
greatest possible precision after research and consultation with various experts. The scenes represented in these photographs are not documented, and if they ever did exist there is no record of them. Nothing is left today but the architectural remains on the premises. These new images are not meant to replace them but to assume a position of apparent veracity, of verisimilitude. Polo's intention here, unlike other artists who work with the fake "found document," is not to play a historical trompe l'oeil, nor to reveal the possibility of manipulating the documentary evidence that configures it. Rather, her photographs are the depiction of a possible history frozen in time, in contrast to the motion of the video that completes the core of her proposal. ...

In the logic of imperialism, Edward Said declared, knowledge is power, and the accumulation of knowledge automatically signifies an accumulation of power. The world maps drawn by the colonial empires are associated with a control that is both symbolic (the act of drawing a map) and real (movement - that of people and the stars-is known and controlled). Faced with the diverse propositions of Apparent Position, there reemerges the image of the unique position that inspired the colonial fever: an empire on which "the sun never set."

Excerpt from a text written by Carlos Martin in occasion of Polo's exbibition at Museo Nacional Reina Sofía. January 2012. 